\title{
A GREAT LAND MARK TO ACHIEVE 4-G MOBILE COMMUNICATIONS
}

\author{
Pijush Kanti Bhattacharjee ${ }^{1}$, Sudipta Roy ${ }^{2}$ \\ ${ }^{I}$ Department of Applied Electronics \& Instrumentation Engineering, Dream Institute of Technology, Kolkata-700104, \\ West Bengal, India \\ ${ }^{2}$ Department of Information Technology, School of Technology, Assam University, Silchar, Pin-788011, Assam, India
}

\begin{abstract}
Celluar mobile communications have brought forth a massive change in telecommunications field due to introducing digital electronics switching and optical fibre as well as microwave and satellite links technologies throught the world. Now people have all communcations facilities and opportunities to step in the super fast mobile communications age with very high speed data communications facility from 2-g to 3-g and henceforth it is building the path from 3-g to 4-g mobile communications also. A great mobile communications revolution happens throughout the world - whose direct impact is that communications in different forms like voice, data, image, conference, multimedia etc. Are avilable for all at any time anywhere with a minimum price.
\end{abstract}

Keywords: PCS, PCN, FCC, AT\&T, VLSI, ULSI, Nano Chips, IMTS, NTT, AMPS, USDC, TACS, NMTS, NAMTS, PDCS, CT2, DECT, FDMA, TDMA, CDMA, GSM, T-S, T-S-T, ISDN, VSAT, SMS, PN, ARFCN, BCCH, BCH, CCCH, DCCH, MS, BTS, BSC, BSS, MSC, HLR, VLR, EIR, AUC, IMSI, TMSI, GMSK, LNA, LAN, WAN, GPRS, EDGE, WCDMA, CDMA-2000, Wi-Fi, Bluetooth, WLAN, WPAN, WCAN, WHAN, MANET, VANET, ITU, ETSI, PDSN, IWF, AAA, HA, FA, RNC, UMTS.

\section{INTRODUCTION TO WIRELESS MOBILE COMMUNICATIONS}

Wireless communication is the most popular area of communications at present. The first wireless communication established by Guglielmo Marconi of Italy in 1897 A.D., when contact between two ships sailing in the English Channel was made through wireless. The development of cellular communications [3]-[14] supports simultaneously voice telephony with other services like transmission of video, images, text and data etc. The two resources are very much limited, i.e., availability of radio spectrum or bandwidth and transmitted power of mobile handset or mobile battery power [6]-[7]. By the invention of new processors (VLSI, ULSI, Nano chips etc.), jelly filled battery and different multiple access technologies (FDMA, TDMA, CDMA etc.), these two problems are solved. There was existing various types of mobile technologies such as Mobile Radio Systems, Satellite Mobile Systems, Cellular Personal Communications Systems (PCSs), Personal Communications Network (PCNs) etc. upto early 1930s was belonging to $1-G$ (First Generation) mobile communications. All mobile radio systems in operation used amplitude modulation (AM). From the late of 1930 onwards (1936 A.D.), Frequency Modulation (FM) systems were implemented in mobile communications. At the end of Second
World War (1946 A.D), the Mobile Telephone Systems (MTS) were extended to commercial purpose in USA; it was introduced in 25 cities. U.S. radio frequency regulatory body known as Federal Communications Commission (FCC) allocates the frequency spectrum for smooth mobile operation. FCC grants license to the American Telephone and Telegraph Company (AT\&T) to operate mobile service in 1946 A.D. This system used carrier bandwidth per channel of $120 \mathrm{KHz}$ in half duplex mode, a single powerful transmitter to cover a radius of 50 miles or more from the base station. In 1950 A.D, the improved technology was enabled to make channel bandwidth to $60 \mathrm{KHz}$. This bandwidth was depended on the Processor's Capacity, RF Filters, Low Noise Amplifier, front-end receiver Amplifiers etc. By the mid of 1960 A.D. in the US, the FM bandwidth of voice transmission was reduced to $30 \mathrm{KHz}$. Improved Mobile Telephone Service (IMTS) has started functioning from 1960 A.D. in the U.S, where the channels were available in full duplex, with extra facilities available like auto dial, auto switching, auto trunking, routing etc.

In 2-G mobile communications, the basic concept of cellular mobile telephone is breaking a coverage zone or area into smaller cells, generally hexagonal region, each of which reuses portions of the spectrum to increase the same frequency spectrum usage. If frequency channels are only reused after $\mathrm{N}$ cells, we recognize the frequency reuse factor as $1 / \mathrm{N}$, then $\mathrm{N}=$ 
$i^{2}+i j+j^{2}$, where $i$ and $j$ are non negative real integers. So, there is a sufficient distance between the same frequency channels, i.e., at least seven cell difference (for $\mathrm{i}=1$ and $\mathrm{j}=2, \mathrm{~N}$ becomes 7) to prevent co-channel interference in GSM system. In 1979 A.D., the World's first cellular system is installed by the Nippon Telephone and Telegraph Company (NTT) in Japan; it uses 600 FM duplex channels $(25 \mathrm{KHz}$ for each one way link) in the 800 $\mathrm{MHz}$ band. A full fledge commercial cellular service is introduced in USA from 1983 A.D., called Advanced Mobile Phone Service (AMPS). AMPS system is an analog system having two frequency band like $825-845 \mathrm{MHz}$ for reverse path (MS to BTS) and $870-890 \mathrm{MHz}$ for forward path (BTS to MS), channel width or bandwidth per channel is $30 \mathrm{KHz}$, for $40 \mathrm{MHz}$ Spectrum where $20 \mathrm{MHz}$ for each path, total number of channels available 666. In the late 1991 A.D., the first US Digital Cellular system (USDC) is standardized by Electronic Industry Association as Interim Standard (IS)-54 and later IS136. It supports three digital channels in the same $30 \mathrm{KHz}$ bandwidth, i.e., the capacity of USDC is three times that of AMPS.

From 1995 A.D. onwards in India, a second generation (2-G) cellular system is started. It is Global System for Mobile Communication (GSM) [3]-[14] as an International Standard by International Telecomm Union, and Code division multiple access (CDMA) system [3]-[14] based on Walsh coding CDMA technique is developed by Qualcomm. Inc and is standardized by the Telecomm Industry Association (TIA) as IS-95. In second generation $(2-\mathrm{G})$ cellular system, the channel capacity is increased by using different multiplexing techniques like Frequency Division Multiple Access (FDMA), Time Division Multiple Access (TDMA), Code Division Multiple Access (CDMA) etc. The forward channel (BTS to MS) and the reverse channel (MS to BTS) are having different frequencies to prevent any interference by Frequency Division Duplexing (FDD) method for simultaneous transmitting and receiving signals. Generally the reverse channel frequency is exactly $45 \mathrm{MHz}$ lower than that of the forward channel to safeguard the communication from interference, noise etc. 2.5-G mobile network is developed under General Packet Radio Service (GPRS) and Enhanced Data Rates for GSM Evolution (EDGE) etc. [7]-[8]; Then 3-G mobile communications are started with Wi-Fi for Wireless Local Area Network (WLAN) and Wide Area Network (WAN) [7]-[10], CDMA-2000 for CDMA and WCDMA for GSM technology [7]-[10] ,i.e., 3-G mobile network are yet to be starting function in full order in the field of mobile communications in India. Now Bluetooth technology (with modem) operating in the $2.4 \mathrm{GHz}$ Industrial Scientific and Medical (ISM) radio Band, i.e., $2400 \mathrm{MHz}$ to $2483.5 \mathrm{MHz}$ is adopted for limited area (say 100 200 meter) wireless network like WLAN or Wireless Personal Area Network (WPAN) [6][14].

Modern digital switches [1]-[2], [6] like T-S (Time-Space), T-S$\mathrm{T}$, T-S-S-T etc. provide huge number of connections from one telephone switching system (exchange) and transmission networks like optical fiber [2], microwave, satellite, co-axial cable network etc. integrate large number of channels through one media with very high speed communication links. Very small aperture terminal (VSAT) using satellite channels are ensuring fast communication to remote places.

\section{EXISTING MOBILE TELEPHONE SYSTEMS USING GSM AND CDMA TECHNOLOGY}

At the end of $20^{\text {th }}$ Century, Licenses for cellular or mobile telephone business [3]-[14] for public use are issued to different private companies in India. In the first phase, the equipment of cellular telephone exchanges and handsets are not reliable. These instruments have various shortcomings, but in no time all type of problems are eliminated with the advancement of technology and research output. The mobile private service is started from 1995 A.D. and from 2000 A.D. BSNL has joined in the mobile scenario of India. Day to day mobile phone is going to be slim miniature size, very light weight, battery long lasting with huge number of facilities and in build memory with camera. At present the mobile telephone is offering multimedia services like simultaneously voice, image, written message, SMS, e-mail, high speed internet, remote control, GPRS, telebusiness etc. Present mobile service is continuing in India of Second Generation (2-G). In Europe, America, Japan and advanced countries, Third generation (3-G) mobile service is running. In 3-G mobile communications, the voice and the data links are quite fast with huge bit rate, e.g., upto 2 Mbps. Thus, the mobile phone (MS) acts as a mini laptop by implementing advance generation mobile communications [6].

Presently we have two types of mobile telephone system in India. First one is GSM or Global System for Mobile Communication [3]-[7], second one CDMA or Code Division Multiple Access system [3]-[8]. Each sector of a hexagonal cell or a BTS [3]-[7] is identified by a unique PN (Pseudo Noise) number or code in CDMA or Absolute Radio Frequency Channel Numbers (ARFCN) broadcasted by Broadcast Control Channel $(\mathrm{BCCH})$ in GSM which indicates the frequency band using, cell, sector and network identity and this PN or ARFCN is transmitted by BTS under regular intervals (say $26.67 \mathrm{msec}$ ). Both GSM and CDMA systems have some merits as well as demerits. By an intricate comparison it can not be assured which one is better than the other. In GSM technology at the transmitter, voice and data (Analog signals digitized to $64 \mathrm{Kbps}$ by PCM) is down sampled by voice coder (Vocoder) at the rate of $13 \mathrm{Kbps}$, multiplexed by Frequency Division Multiple Access (FDMA) method by RF carriers and then the FDMA signals are further multiplexed by Time Division Multiple Access (TDMA) technique [4]-[7] into 8 time slots (TS) per carrier, finally transmitted by Gaussian Minimum Shift Keying (GMSK) modulation technique. At the receiver, reverse process take place for recovering the basic signals. Two types of GSM logic channels are available, called Traffic channels (TCHs) and Control channels (CCHs). Traffic channels carry digitally encoded speech or data with identical functions and formats on 
both forward and reverse path. Control channels carry signaling, synchronization etc. between BTS and MS in both links. There are six different types of TCHs and large number of CCHs. Full rate Speech Channel (TCH/FS) carries user speech digitized at the rate of $13 \mathrm{Kbps}$, with coding and error correction bits (control bits) added is increased to $22.8 \mathrm{Kbps}$. Three main control channels are available like Broadcast Channel $(\mathrm{BCH})$ carry identity (ARFCN), Common Control Channel (CCCH), Dedicated Control Channel (DCCH).

CDMA system architecture follows the same pattern like that of GSM, but interfaces and protocols are different from GSM system. In CDMA technique at the transmitter, the basic signals, i.e., voice and data (64 Kbps by PCM) are down sampled at the rate of $8.5 \mathrm{Kbps}$ or $13 \mathrm{Kbps}$ by Vocoder, multiplexed by FDMA technique with the carrier frequency (FA), then the each multiplexed carrier signal (FA) is further modulated by CDMA Walsh coding in different number of codes (e.g. 64 codes, each having 64 bits length and these codes are orthogonal to each other) adjoining with PN code according to the capacity of exchange and other parameters like error checking, encrypting, control bits etc. At the CDMA receiver, the basic signals are reconstructed by reverse and demodulation process with Low Noise Amplifier (LNA) and Filters, which curtail all sorts of noises and make good for distortion of the original signal. For centralized control in a mobile network, all MSCs are connected to a central MSC like mainframe or super computer called NOC (Network Operating Center) which keeps all information, databases of the network. Hence NOC controls the total network from a particular place, where it is situated. Five important technical parameters [3]-[14] at any place such as Transmitted Signal Power Level $\left(T_{x}\right)$, Received Signal Power Level $\left(R_{x}\right)$, Signal to Noise Ratio $\left(\mathrm{E}_{\mathrm{c}} / \mathrm{I}_{0}\right.$ in CDMA, C/I in GSM), Frame Error Rate (FER), Received Signal Quality ( $\mathrm{R}_{\mathrm{x}}$ Qual in GSM) determine the quality of signal or speech transmitting or receiving by the MS (Mobile Subscriber or Station) and the BTS at that place. By this coverage of signals from a BTS or a cell are determined, so that good quality of speech or data is provided throughout the region and no blind zone, i.e., low quality received signal exists. The standard $R_{x}$ (Receive Power) level should be higher than $-95 \mathrm{dBm}$ and FER should not be more than $2 \%$ for stable reliable communication. These parameters decide a cell or a BTS area and a call hand over or hand off situation with the other cell or the other sector. The packet switched can be enhanced in different speeds in 2-G and 3-G mobile communications such as $38.4 \mathrm{kbps}, 76.8 \mathrm{kbps}, 153.6$ kbps etc.

2-G mobile communications is upgraded to $2.5-\mathrm{G}$ stage by deploying either General Packet Radio Service (GPRS) or Enhanced Data Rates for GSM Evolution (EDGE) technology. GPRS is originally standardized by European Telecommunications Standards Institute (ETSI). EDGE is standardized by $3^{\text {rd }}$ Generation Partnership Project (3GPP) in 2003 A.D. Data rates safely used in 2.5-G mobile communications is from 400 Kilo bits per sec (Kbps) to 1 Mega bits per sec (Mbps).

\section{COMPARISON BETWEEN GSM \& CDMA}

\subsection{GSM-900 Frequency Band System}

$890-915 \mathrm{MHz}$ for uplink or reverse link from MS to BTS $935-960 \mathrm{MHz}$ for downlink or forward link from BTS to MS

Bandwidth $=(915-890) \mathrm{MHz}=25 \mathrm{MHz}$.

R.F. carrier channel spacing, i.e., Frequency difference between two adjacent channels $=200 \mathrm{KHz}$.

Total number of R.F. carrier by FDMA $=124(100 \mathrm{KHz}$ guard band is given in two sides, i.e., at starting and ending of a frame)

Number of Time Slots (TS) by TDMA in One R.F carrier, i.e., Voice channel per carrier $=8$

Total number of Time Slots $(\mathrm{TS})=124 \times 8=992$

[If one BTS has 12 R.F. carrier frequency in a cell, i.e. it contains $=12 \times 8=96$ TS $]$.

BTS Power transmitting $=$ Max 40 Watt.

MS Power transmitting $=200 \mathrm{mWatt}$ to $2 \mathrm{~W}$.

\subsection{GSM-1800 Frequency Band System}

$1710-1785 \mathrm{MHz}$ for uplink or reverse link from MS to BTS $1805-1880 \mathrm{MHz}$ for downlink or forward link from BTS to MS

Here the number of channels is increased by taking wide spectrum, i.e., Bandwidth $75 \mathrm{MHz}$, and applying with FDMA and TDMA multiplexing techniques. International Telecommunications Union (ITU) recommended this spectrum for GSM-1800 MHz band, but the transmitter and the receiver instrument become costly and interferences are more due to adopting high frequency signals.

\subsection{GSM and CDMA System Architecture}

GSM and CDMA system architecture block diagram is described in Fig. 1. GSM system consists of following components:

MS - Mobile Station or Mobile Telephone for transmitting and receiving signals.

MS to BTS path - Reverse or Up link, BTS to MS path - Forward or Down link.

BTS - Base Transceiver Station serves mobile connection to one or more cells and sectors in the cellular network, contains TRXs, i.e., transceivers or radio units.

BSS - Base Station Sub System consists of BSCs \& BTSs.

BSC - Base Switching Center controls one or more BTSs and perform inter BTS and intra BTS switching and handovers. 
MSC - Mobile Switching Center or Main Switching Center which is a basic digital electronics exchange, e.g., 5ESS, i.e., $5^{\text {th }}$ version of Electronics Switching System. It controls all the functions of a Mobile Network.
HLR - Home Location Register occupies identities of mobile subscriber as IMSI [International Mobile Subscriber's Identity], service parameters, location information etc.

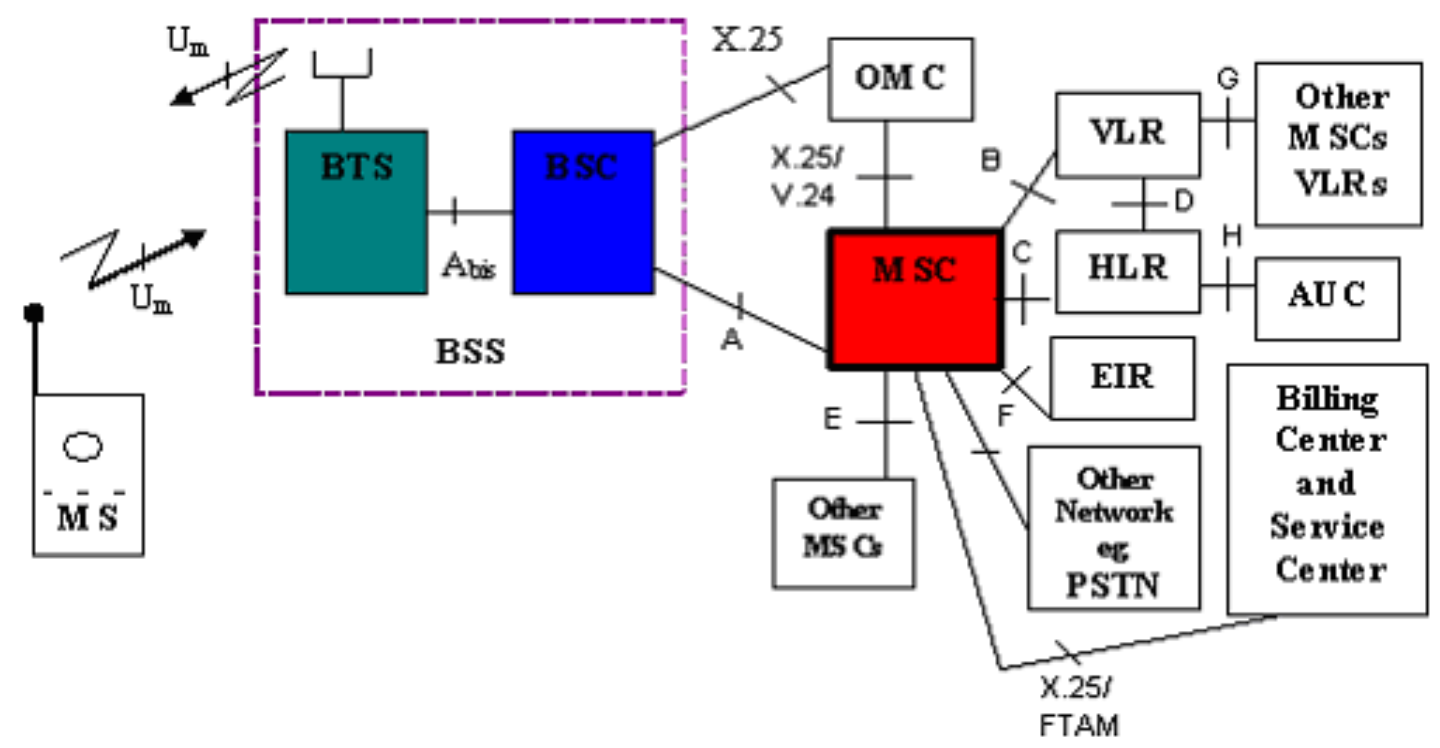

Fig 1 GSM and CDMA System Architecture

VLR - Visitor Location Register contains permanent and temporary (roaming) mobile subscriber's identity as TMSI, ISDN directory number, routing etc.

EIR - Equipment Identity Register contains identity of mobile equipment called IMEI [International Mobile Equipment Identity]. It may be valid, suspect or prohibited.

AUC - Authentication Center contains authentication data called $\mathrm{K}_{\mathrm{i}}$ for encrypting user speech and data security purpose.

OMC - Operation and Maintenance Center is centralized maintenance to operate, administer and monitor the functions of MSCs, BSSs etc. One OMC may control number of MSCs and BSSs. OMC is having a mainframe computer.

Billing Center - It provides all sorts of charging or commercial information. One billing center can handle the calls from several MSCs.

Service Center - It assures special services like SMS, e-mail etc. Interfaces $-\mathrm{U}_{\mathrm{m}}$ (Air interface- $22.8 \mathrm{Kbps}$ ), $\mathrm{A}_{\text {bis }}(16 \mathrm{Kbps}), \mathrm{A}$ (64 Kbps), B, C, D, E, F, G, H, X.25, FTAM, V.24.

Presently CDMA system has gain more popularity over the GSM System due to some sorts of advantage and usability obtained from CDMA system. Moreover number of channels in a cell or a sector in the CDMA system is more from a BTS due to same frequency reusing possible in the adjacent cell or sector, which offers more connections to the subscribers encouraging the service provider's point of view.

\subsection{CDMA Frequency Band System}

$824-844 \mathrm{MHz}$ for uplink or reverse link from MS to BTS $869-889 \mathrm{MHz}$ for downlink or forward link from BTS to MS

R.F. carrier channel spacing $=1.25 \mathrm{MHz}$,

Total available Bandwidth $=20 \mathrm{MHz}$.

Total number of carriers $(\mathrm{FAs})=16$

Voice channel per carrier $($ CDMA $)=35 \sim 40$ traffic channels It uses 64 number of Walsh orthogonal codes, each code having 64 bits length. Walsh orthogonal code means that if EXOR functions are taken between two adjacent orthogonal codes, output of the EXOR function will be zero.

Total number of CDMA Channels $=40 \times 16=640$

BTS Power transmitting = Max 20 Watt.

MS Power transmitting = Max 200 mWatt.

CDMA system has large capacity comparing to other techniques like GSM. It requires less power using spread spectrum technique [3]-[7], [12]-[14]. Spread spectrum technique is adopted by multiplying Walsh code with Pseudorandom Noise (PN) Code, since one carrier channel is having large bandwidth 1.25 Mz. There are two types of PN codes, One is long PN code 
having $2^{42}$ combinations (4400 billion) using 42 bits length, unique PN long code is given to each MS for identifying IMSI, ESN etc., repeated every 41 days and the other is PN short code which uses $2^{15}$ combinations (32768) using 15 bits length. Unique PN short code is assured to each BTS for identifying cell or sector number, network parameters etc., repeated every $26.67 \mathrm{msec}$. The BTS antenna of CDMA requires to transmit maximum 20 Watt power where as GSM antenna transmits maximum 40 Watt power and the mobile handset of CDMA transmits maximum $200 \mathrm{mWatt}$ whereas the same of GSM transmits maximum 2 Watt.

\section{ARCHITECTURE OF 3-G MOBILE SYSTEM}

Architecture of a $3^{\text {rd }}$ Generation wireless network CDMA-2000 or WCDMA is described below in Fig. 2. This 3-G network [8]-[10] can provide circuit switched voice service, circuit switched data service like 2-G (CDMA One or GSM) in addition to this packet switched data and multimedia service. In 2000 A.D., Telecommunication Industry Association (TIA) publishes Interim Standard (IS)-856 network. It is known CDMA 2000 1X EV-DO (Evolution Data Optimized). CDMA$20001 \mathrm{X}$ is having chip rate $1.2288 \mathrm{Mcps}$, While WCDMA chip rate is $3.84 \mathrm{Mcps}$, but CDMA-2000 $3 \mathrm{X}$ chip rate is 3.6864 Mcps.

In data network, MS is called Access Terminal (AT) where data or messages in written form is originated or terminated, where as BTS with BSC are called Access Network (AN) which handles data and further transports to PDSN (Packet Data Serving Node) through Interim Standard-2001 (IS-2001) network specified by ITU. Thus AN acts as an interface between AT and PDSN. AT and AN are connected by IS-856 network.

For increasing data rate in 2-G, the first step begins with deploying GPRS or the Public Switched Data Network (PSDN) for enabling packet data services in GSM and CDMA-One networks. The Voice-over-IP (VoIP) gateway function could be provided as an extended feature to the circuit gateway or the PDSN for 3-G mobile communications. The VoIP gateway will hold the vocoding algorithms converting between a voice call encapsulated in an air interface frame and an IP end point that may be an IP-enabled phone, IP based PBX or PC etc. [7]-[14].

The circuit switched voice and data services are arranged in same pattern as CDMA-One (2-G) by MS, BTS, BSC, MSC, HLR, VLR, AUC and IWF. An Inter-Working Function (IWF) is configured for converting a signal into a form compatible with a destination network receiving the data. While IWF enables circuit switched data service and BSC carries out mobility management, i.e., controlling hand over or hand off. Additional networks are provided in 3-G for providing packet switched data service usually higher speed than that of circuit switched data service in $2-\mathrm{G}$.
This packet switched data network [8]-[10] is consisting of two parts.

(1) Packet Data Serving Node (PDSN): The PDSN is the element that provides packet switched data service, like MSC for circuit switching. It is an internet protocol (IP) router that switches user data traffic to a public data network, i.e., the internet. It deals with packet switched traffic (generally data) between the MS, i.e., the user and packet switched network namely Internet or Intranet etc.

(2) Authentication, Authorization and Accounting (AAA): The AAA is a server that provides three main functions like authentication, authorization and accounting services for the packet data traffic connected with PDSN. It ultimately ensures packet data network connectivity services to the mobile users. Authentication requires the user to provide an account number and password, i.e., exchange of logical keys or certificates between the client and the server. If this authentication is correct, the MS is permitted for packet data service by Authorization. Last but not the least, function of AAA is accounting. It collects information on its usage of packet data service for billing or tariff calculation.

The CDMA-2000 network is supporting simple IP and mobile IP functions.

(i) Simple IP: An MS residing in home PDSN is given an IP address $\mathrm{M}$ and the server on the internet has an IP address $\mathrm{S}$. Using these two addresses, IP packets containing data or information are exchanged between the MS and different servers in the same PDSN. A PDSN is consisting of several servers for routing packets in different directions. These servers are identified by the assigned address.

(ii) Mobile IP: Two additional network elements are provided for supporting Mobile IP.

(a) Home Agent (HA): This is a router together with the foreign agent (FA). This router resides on the MS home IP network. It serves as a point for communications with the mobile network.

(b) Foreign Agent (FA): This is another router residing in other PDSN. When MS travels a foreign IP network, the FA in the foreign network receives packet forwarded from the HA and delivers them to the MS. Thus it functions as the mobile node's point of attachment when it travels to the foreign network, i.e., the network other than its home network.

Thus mobile IP uses a tunneling protocol to allow messages from the PDSN to be directed to the mobile node's IP address. This is accomplished by way of routing messages to the foreign node for delivery via tunneling the original IP address inside a packet destined for the temporary IP address assigned to the mobile node by the foreign node. This method allows for seamless communications between the mobile node and applications residing on the PDSN, always-on connectivity for mobile data applications and wireless computing. There are three modes of operation for WCDMA or CDMA-2000

- Direct sequence (DS) WCDMA in UMTS for frequency Division Duplex (FDD).

- W-CDMA Time Division Duplex (TDD). 
- $\quad$ CDMA 2000 Multicarrier FDD.

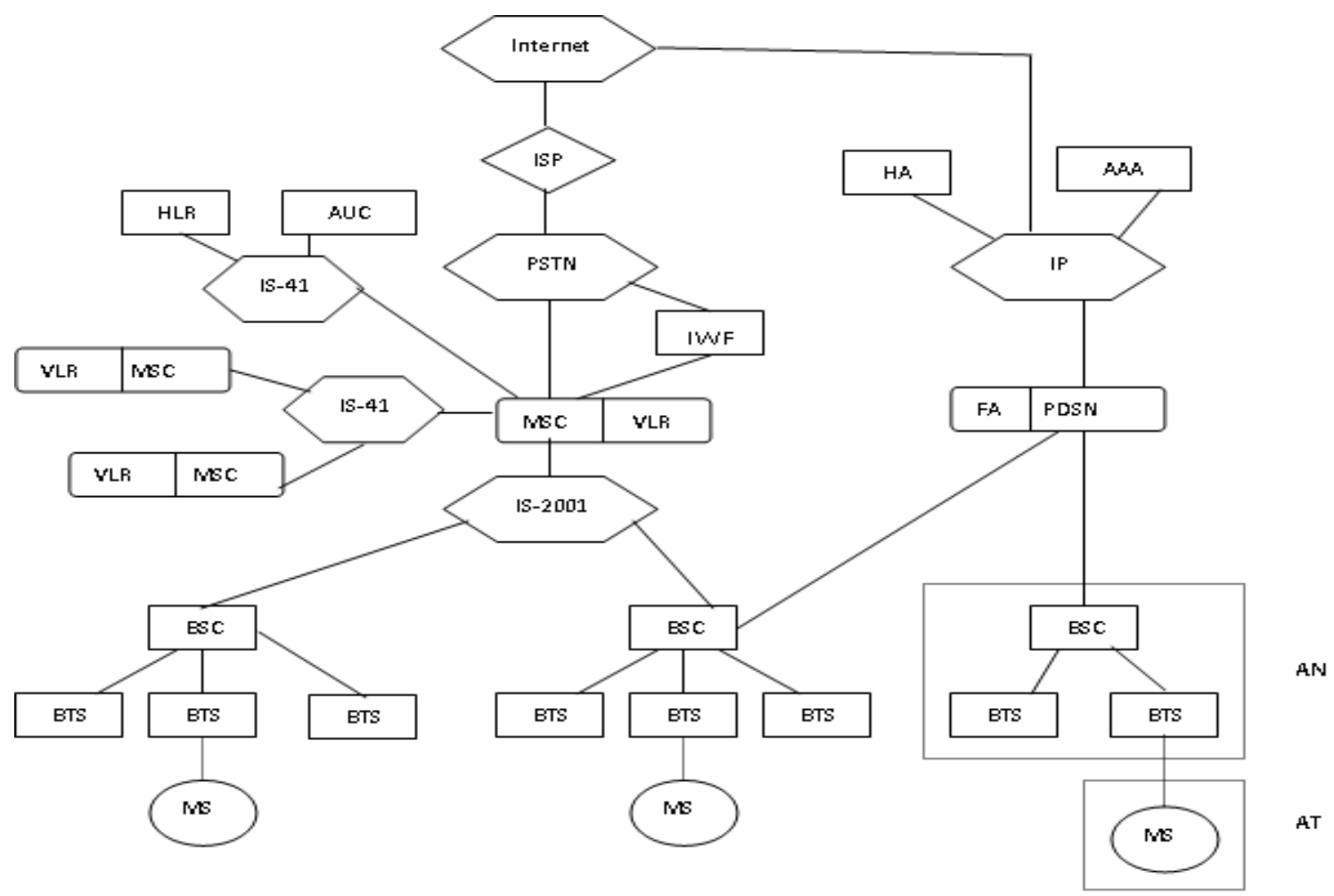

Fig. 2 A typical 3-G wireless network architecture with high speed data network

\section{ARCHITECTURE OF 4-G MOBILE SYSTEM}

4-G mobile communications [11]-[12] are under experimental stage and it is not yet totally developed. We have designed the architecture of a fourth generation (4-G) wireless network as shown in Fig. 3. This 4-G network can provide circuit switched voice service, circuit switched data service like 2-G (CDMAOne or GSM), 3-G (WCDMA, CDMA-2000, UMTS), in addition to this packet switched data and multimedia service at a very high data rate [6]-[14]. Data rate dimensioning targets for 4-G is 50 to $500 \mathrm{bit} / \mathrm{s} / \mathrm{Hz} / \mathrm{Km}^{2}$, whereas in $3-\mathrm{G}$ it is around 10 $\mathrm{bit} / \mathrm{s} / \mathrm{Hz} / \mathrm{Km}^{2}$ using High Speed Downlink Packet Access (HSDPA) technology. Now we mention some abbreviations and briefly describe them that are used in 4-G mobile communications technology.

RNC - Radio Network Controller in Universal Mobile Telecommunication Service (UMTS) in 3-G, 4-G, like BSC in GSM or CDMA in 2-G and 3-G.

Access controller provides connection to user's network with server or switch.

Service adaptation or gateway - It extends connection to other world wide network or Internet.
PSTN - Public Switched Telephone Network, i.e., land or wire line telephone network.

ISDN - Integrated Services Digital Network

PDN - Public Data Network

Server - AAA server, or PDSN server, or any other server for high speed data communications, in general, but in special conditions it can also provide voice communications.

Switch - It is used to give circuit connectivity like circuit or packet or both switching system for voice and data communications.

Workstation - It is used for connecting data network or any other network consisting of computers (PCs, Laptop, etc.), mobile phones, etc. 


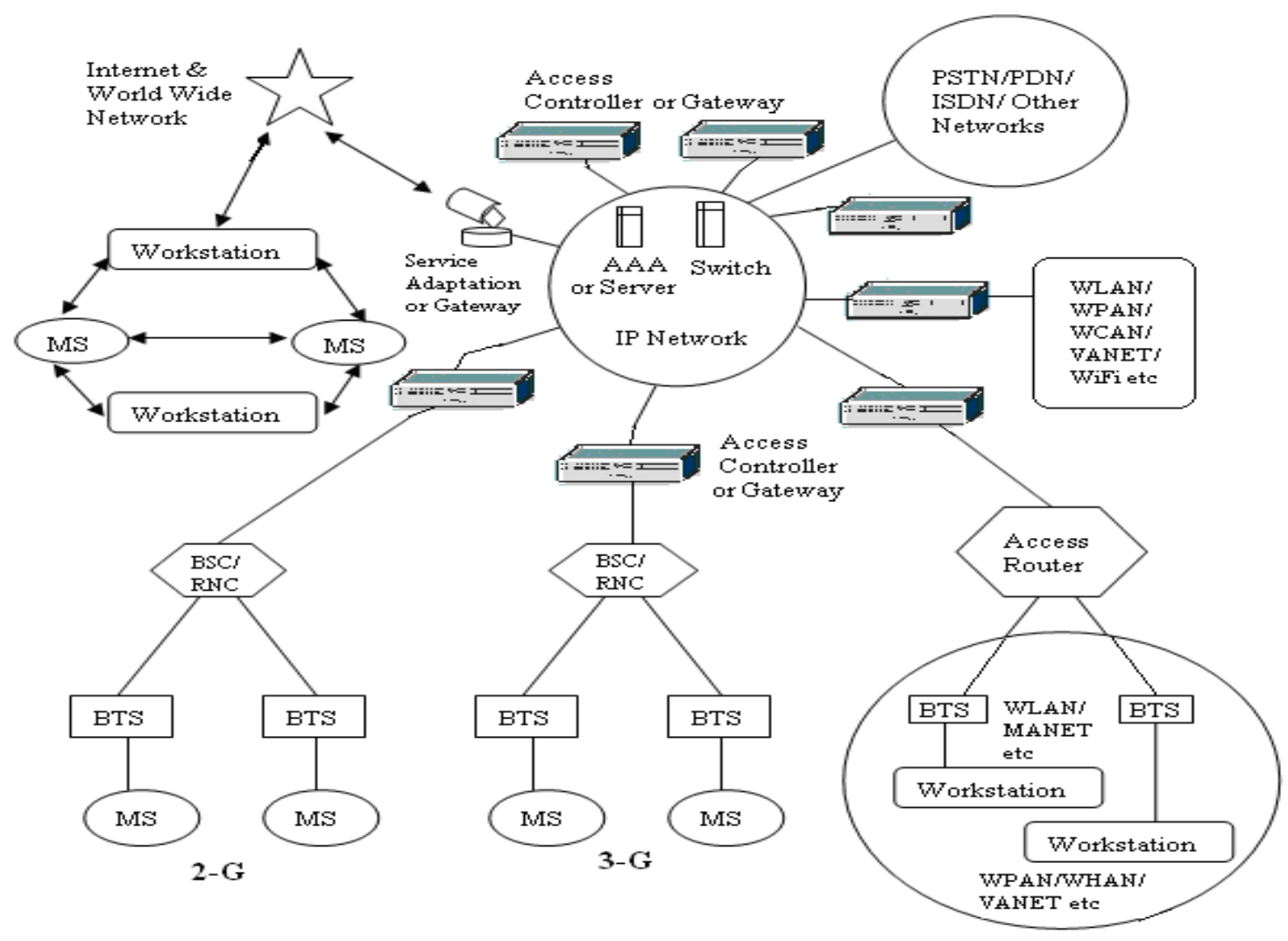

Fig 3 Wireless network architecture for fourth generation (4-G) mobile communications

In 4-G mobile communications, additional network is provided that Access Controller or Gateway for the network is connected from the switch or server in between the switch or server to user's equipment or Internet or other networks. In essence, 4-G aims to transfer communications architectures from traditional vertical stovepipe to horizontal integrated systems [11]-[14]. Personal Networks like Wireless Local Area Network (WLAN), Wireless Personal Area Network (WPAN), Wireless Corporate Area Network (WCAN), Wireless Home Area Network (WHAN), Wireless Fidelity (Wi-Fi), Worldwide Interoperability for Microwave Access (WiMAX), Mobile Ad Hoc Network (MANET), Vehicular Ad Hoc Network (VANET) etc. are a dynamic network building on the above mentioned wireless networking technologies, which facilitate personalized communications with numerous number of subscribers anywhere at any time.

WiMAX provides speed of communications through wireless upto $40 \mathrm{Mbps}$ and it can be extended upto $1 \mathrm{Gbps}$, in a radius of $50 \mathrm{Km}$ area. Thus all the subscriber's networks are connected to a 4-G mobile system, since the personal networks can be expanded or shrunken depending on the availability of users, their demands and environment. A WPAN is a network of devices that consist of a mobile phone, a personal digital or data assistant (PDA), a notebook PC, a digital camera, etc. The
WPAN expansion can physically be made via interconnecting structures, e.g., GSM and CDMA 2-G, 2.5-G General Packet Radio Service (GPRS), Enhanced Data Rates for GSM Evolution (EDGE), 3-G mobile communications (WCDMA, CDMA-2000, UMTS, etc.) and the Internet, to remote networks such as home area networks, corporate area networks or vehicular area networks, etc. in 4-G mobile communications.

\section{CONCLUSIONS}

From first generation (1-G) to fourth generation (4-G) mobile communications development is completely described in this paper in a new measure. It has been observed that the wireless communications are employed packet switching technology in 3-G and 4-G in full order with utilizing Internet Protocol (IP) network, i.e., IPv4 or IPv6 network; as a result high speed secured data as well as voice transmission-reception is possible throughout the world and universe. Although 4-G mobile communications is under experimental stage at present and it is not yet marketed, but the proposed design of 4-G mobile communications are made such that it can provide very fast multimedia communications in ubiquitous access. 


\section{REFERENCES}

[1]. J. Dunlop and D. G. Smith, Telecommunication Engineering, Chapman \& Hall Publishers, $3^{\text {rd }}$ Ed, 1994.

[2]. J. M. Senior, Optical Fiber Communications- Principles and Practice, Prentice Hall Publisher, $2^{\text {nd }} E d, 1992$.

[3]. A. J. Viterbi, CDMA Principles of Spread Spectrum Communication, Addison-Wiseley Publishers, 1995.

[4]. I. Koutsopoulos, L. Tassiulas, "The impact of space division multiplexing on resource allocation: a unified treatment of TDMA, OFDMA and CDMA", IEEE Transaction on Communication, vol. 56, no. 2, pp. 260-269, 2008.

[5]. M. Rahnema, "Overview of the GSM System and Protocol Architecture", IEEE Transaction on Communications, pp. 92100, April 1993.

[6]. P. K. Bhattacharjee, "A New Era in Mobile Communications- GSM and CDMA", National Conference on Wireless and Optical Communications (WOC-07) at Punjab Engg College (D.U), pp 118 - 126, Dec 2007.

[7]. S. Rappaport, Wireless Communication: Principles and Practice, Prentice Hall Pub Ltd, $2^{\text {nd }}$ Ed, 2006.

[8]. Xiaodong Wang and H. Vincent Poor, Wireless Communication Systems, Pearson Education Pvt Ltd, New Delhi, First Indian Rept, 2004.

[9]. C. Koner, P. K. Bhattacharjee, C. T. Bhunia, U Maulik, "A Novel Approach for Authentication Technique in Mobile Communications", International Journal of Computer Theory and Engineering, Singapore, vol. 1, no. 3, pp. 225-229, 2009.

[10]. William C. Y. Lee, Wireless and Cellular Communications, 3rd Edition, McGraw Hill Publishers 2008.

[11]. M. L. Roberts, M. A. Temple, R. F. Mills, and R. A. Raines "Evolution of the Air Interface of Cellular Communications Systems toward 4G Realization", IEEE Communications Surveys and Tutorials, vol. 8, no. 1, pp. 2-23, Mar 2006.

[12]. P. K. Bhattacharjee, "Artificial Intelligence Based Mutual Authentication Technique With Four Entities In 4-G Mobile Communications", International Journal Recent Trends in Engineering, Academy Publishers, Finland, vol. 3, no. 2, pp 2931, May 2010.

[13]. P. Ramjee, O. Tero, "An Overview of CDMA Evolution towards Wideband CDMA", IEEE Communications Survey, 1998.

[14]. F. Adachi, M. Sawahashi, H. Suda, "Wideband DSCDMA for Next Generation Mobile Communications System", IEEE Communication Magazine, pp. 56-69, Sep. 1998. 